\title{
Investigation of ultrasonic-assisted CNC cutting of honeycomb cores
}

\author{
Dongfang $\mathrm{Mu}^{1} \cdot$ Xiaoping $\mathrm{Hu}^{1} \cdot$ Haofeng $\mathrm{Yu}^{1} \cdot$ Baohua $\mathrm{Yu}^{1}$
}

Received: 13 January 2021 / Accepted: 26 July 2021 / Published online: 6 August 2021

(C) The Author(s) 2021

\begin{abstract}
As compared to traditional milling, ultrasonic-assisted cutting of honeycomb core materials has the advantages of small cutting force and superior surface quality. Currently, ultrasonic straight blade V-shaped machining is one of the most efficient roughing processes. The requirement of a processing path to be post-processed based on the processing characteristics of the straight blade cutter is one of the key issues faced while meeting the technological requirements of CNC machine tools for V-shaped machining. A post-processing method of $\mathrm{NC}$ code for $\mathrm{V}$-shaped machining of straight blade cutter is proposed in this article. Initially, the V-shaped rough machining process planning is done, followed by the usage of Unigraphics (UG) to output the initial CNC code. Next, the CNC code is executed through the three key technologies of the second arrangement of cutter locations. Later, research is done on information planning of lifting and lowering cutter, along with research on planning of chip breaking toolpath. Eventually, the simulation cutting experiment is carried out through VERICUT. The results demonstrate that the postprocessing method can meet the processing requirements.
\end{abstract}

Keywords Ultrasonic-assisted cutting $\cdot$ Honeycomb cores $\cdot$ Straight blade cutter $\cdot$ Post-processing

\section{Introduction}

In recent years, the automotive, aerospace, and defense industries have started choosing honeycomb cores as the core material for various high-performance and energy-absorbing structural components. Honeycomb cores are known to have superior physical properties such as high specific strength, high specific stiffness, exceptional fatigue resistance, low relative density, and corrosion resistance $[1,2]$. The traditional milling process will lead to poor surface quality [3] and material dust pollution problems [4] because of the characteristics of orthotropic and heterogeneous structures of honeycomb cores. Ultrasonic-assisted cutting has the potential to reduce the cutting force, make intermittent contact between the tool and the workpiece, reduce tool wear, and effectively overcome the shortcomings of traditional milling. To improve the process planning of processing honeycomb parts and to promote the application and research of ultrasonic machining

Xiaoping $\mathrm{Hu}$

xiaoping.hu@hdu.edu.cn

1 School of Mechanical Engineering, Hangzhou Dianzi University, Hangzhou 310018, China technology in the field of processing materials with weak rigidity such as honeycomb cores, the research on the numerical control machining of ultrasonic-assisted cutting honeycomb cores is of great significance.

For ultrasonic-assisted cutting, a straight blade cutter and disc cutter are used. The cutting forms and methods of these two cutters are completely different from that of the traditional milling cutters. Because of the thin blades of these cutters, a single pass, which is enough to separate the material on both sides of the blade, cannot make the material fall off. Also, the technological characteristics of thin-edged cutters are different from the traditional tools. To obtain a tool path with a small cutting depth and regular chips and to improve the cutting efficiency, modification of the tool path of odd and even layers must be done by planning the order of cutter location (CL).

Post-processing technology refers to converting the planned tool path into a code that can be recognized by the machine tool control system. This code can be used for machine tool numerical control simulation or machine tool processing [5]. At present, post-processors provided by several softwares can be customized for specific model machine tools to develop critical processors for the machine tools [6]; the post-processor needs to be independently developed when the general-purpose post-processor cannot meet the user requirements. Recently, the post-processing algorithms of five-axis 
machine tools with different structures are being developed by researchers. $\mathrm{Li}$ [7] and the team took the machine tool structure of the Hamer C20U model five-axis vertical machining center as an example and successfully developed a five-axis post-processor for Heidenhain dual-turntable based on UG6.0 and verified the simulation. A method for customizing the post-processor of a five-axis machine tool based on Edgecam programming was proposed and verified by Li [8]. Hao [9] and others proposed a custom modification for the post-processing of Master CAM software for AC double-swinging five-axis machine tools and verified it by considering the example of five-axis drilling. To solve the problem of post-processing of a five-axis machine tool with dual rotary tables from workpiece coordinate system to machine tool coordinate system, a solution method called Two Circle Spherical method (TCS) was proposed by $\mathrm{Yu}[10]$.

A new BIUC (Blade-Inclined Ultrasonic Cutting) method for cutting a Nomex honeycomb core with a straight blade was proposed by Wang et al. [11]. As compared to the ultrasonic cutting, BIUC has an extra rotation angle to move the major flank away from the machined surface. By this method, the geometric interference between the major flank and the machined surface can be reduced, which helps to reduce the deformation of the machined side and avoid crushing. Nonetheless, this method has higher requirements for tool path planning and $\mathrm{CNC}$ programs. Cui et al. [12] proposed a path planning and generating method for V-shaped robotic cutting of Nomex honeycomb by straight blade tool. By this, the use of disc tools for surface cleaning between each cutting layer can be avoided, and the machining efficiency can be improved. There is a lack of discussion of the situation that needs to be turned on the workpiece in the path planning and the treatment of the unbroken chips on the workpiece. A new planning method and the corresponding post-processing method in response to the above problems have been proposed in this article.

In this paper, the $\mathrm{CNC}$ cutting process of ultrasonicassisted machining has been introduced in Section 2, along with rough machining process planning and finishing process planning. Section 3 describes the key technology of ultrasonic-assisted cutting CNC programming. Unigraphics (UG) is used for preliminary process planning, followed by post-processing planning. In Section 4, the effectiveness of this method is illustrated with a cutting simulation experiment based on VERICUT.

\section{Analysis of NC cutting process of typical parts}

The application of honeycomb cores in the aerospace field includes wings and spoilers. The surface of honeycomb parts is usually a curved surface with small curvature and smooth changes [13]. An example part is displayed in Fig. 1. Flat, inclined, convex, concave, and other features are demonstrated in this part. It is a typical honeycomb part preferred in the aviation manufacturing field.

\section{1 "V"-shaped process for rough machining}

According to the research mentioned in the existing literature [14], the "V" processing technology is preferred for rough machining. As highlighted in Fig. 2, a straight blade cutter cuts twice with the same swing angle and inclination angle (in the tool coordinate system), and the cutting surface is formed into a "V" shape (triangle and diamond chips). Because of high cutting efficiency, this cutting method is often used in the roughing process.

Ultrasonic-assisted cutting uses a straight blade cutter. Also, its process planning is quite different from the traditional tool, especially the selection of procedures and the setting of processing parameters such as step and cutting depth are different from the conventional settings. The key steps of "V" shape roughing process planning are as follows:

(1) Select cavity milling as the processing procedure;

(2) Equivalent replace cutter, which is equivalent to a slender cylinder milling tool, as shown in Fig. 3;

(3) Set cutting parameters: (a) step distance, cutting depth, $f=2 d \sin \alpha+e / \cos \alpha, a_{p}=\frac{d}{\sqrt{\tan ^{2} \alpha+\frac{1}{\sin ^{2} \theta}}}$; where $d$ is the axial cutting depth of the tool, $\alpha$ is the yaw angle, $\theta$ is the rake angle (as shown in Fig. 4), and $e$ is the blade thickness (refer to Fig. 3); (b) the margin is set to $0 \mathrm{~mm}$; and (c) to align the starting tool paths of the upper and lower layers, the starting point of cutting is set at the corner with the largest vertical cutting amount of the blank.

\subsection{Finishing process planning}

The finishing process of ultrasonic-assisted cutting primarily includes the disc cutter finishing process. UG can be used for complete process planning since the cutting form of the disc cutter is rotary motion, and its high-frequency vibration along the cutter axis has little relevance to the process planning method. Its key technology is elaborated below:

(1) Select variable contour milling as the processing method;

(2) Equivalent replace cutter - set the disc cutter to the same diameter cylinder milling tool; the height of the cylinder is slightly larger than the cutting thickness, as shown in Fig. 5; 
Fig. 1 Honeycomb parts with typical features

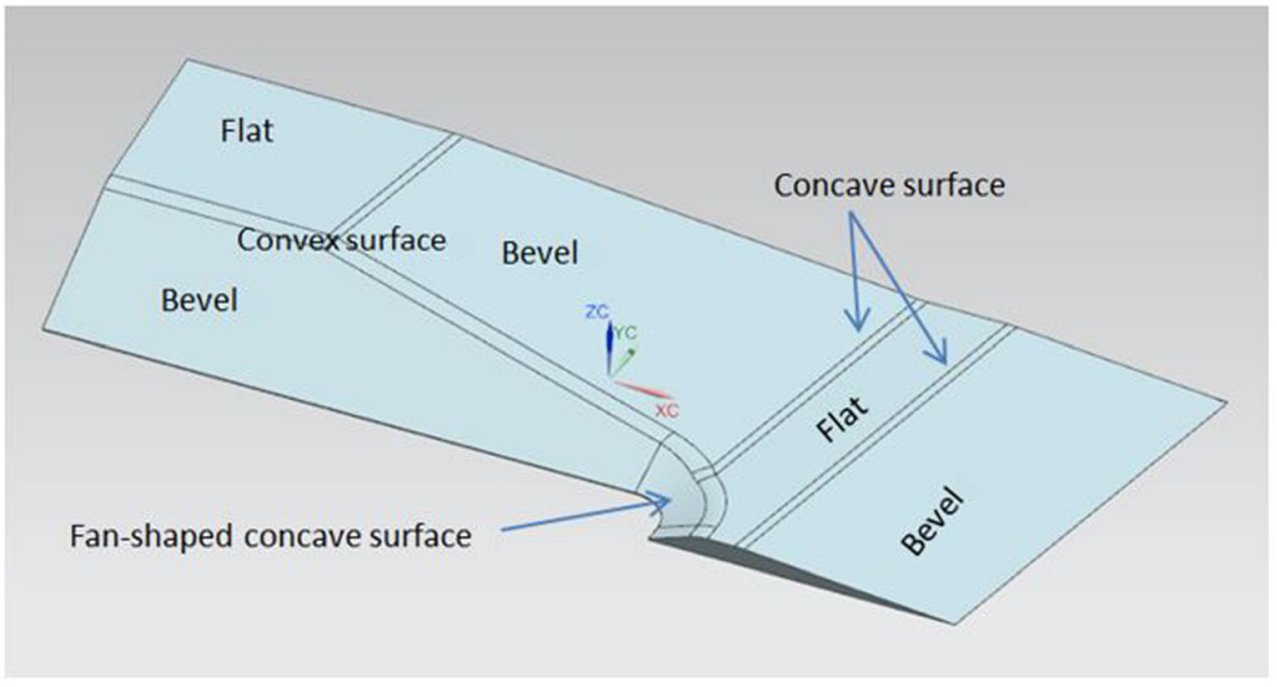

(3) Select the driving of the surface area as the driving method and carry out independent planning based on different surface features of the part surface;

(4) Cutting angle - set a smaller rake angle and yaw angle to reduce the generation of cutting heat.

\section{The key technology of ultrasonic-assisted cutting CNC programming}

For the "V"-shaped rough machining process, use UG for preliminary process planning and then post-processing planning. The initial code output by UG based on MATLAB compiler software is adapted. During the finishing process, UG is directly used to plan the path for the ultrasonic disc cutter.
Eventually, a CNC program for machining is generated using UG.

\subsection{Second arrangement of CLs}

In the "V"-shaped machining process, the tool path between the odd and even layers are misaligned by $0.5 \mathrm{f}$. This helps to obtain a tool path with a small cutting depth and uniform chip formation, forming a diamond-shaped cross-section.

To form the odd and even layer cutting in the second layout process, the tool path of the odd or even layer is translated in the $Y$ direction. Then the single cutting can only realize one of the "V"-shaped edges in the "V"-shaped processing technology, and the other side needs to be cut on one side of the layer after changing. Change the straight blade cutter swing angle and again cut along the same tool path, as demonstrated in Fig. 6b, c. This will lead to a Y-empty situation.
Fig. 2 Ultrasonic straight blade cutter and disc cutter

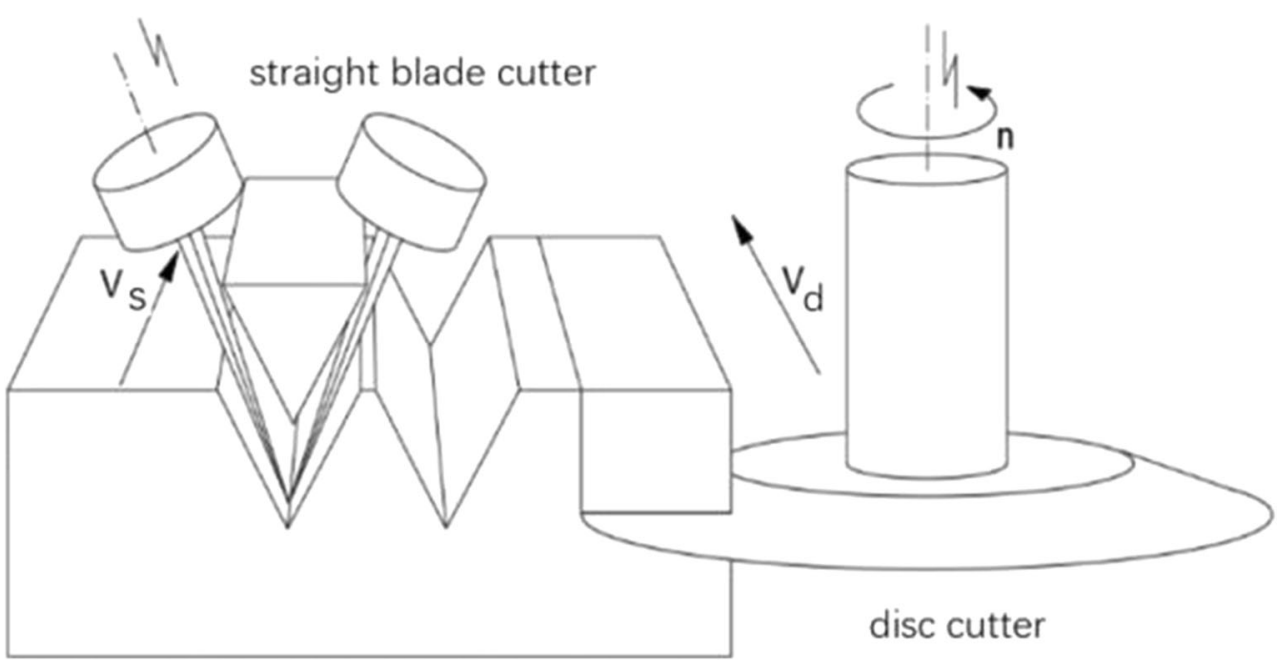


Fig. 3 Equivalent replace ultrasonic straight blade cutter. a Straight blade cutter. b Cylinder milling tool

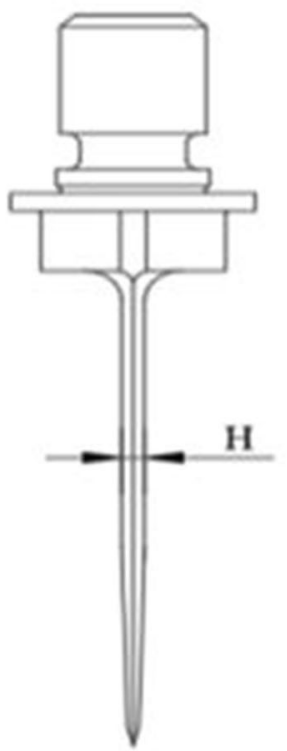

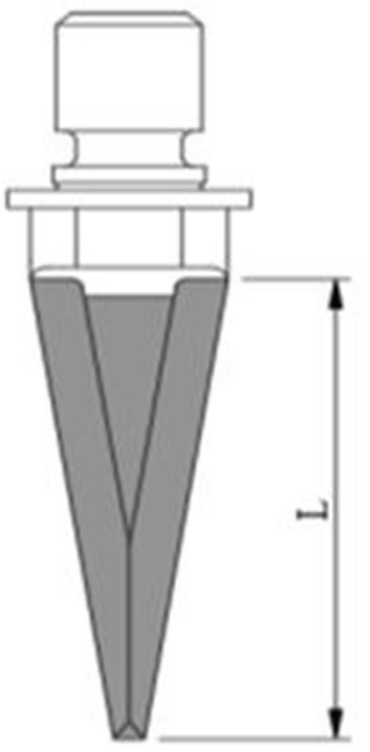

a

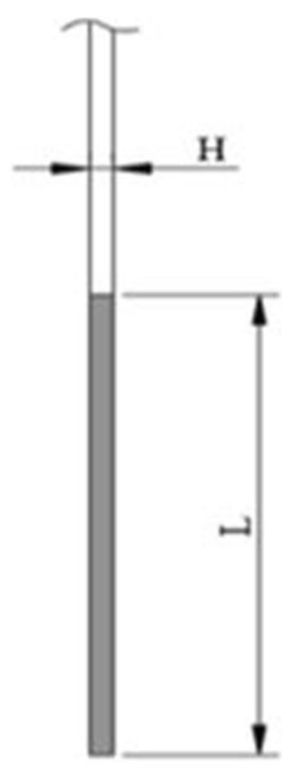

b
As shown in Fig. 7a, the step distance is set to $0.5 \mathrm{f}$. In the second layout process, CL of the odd and even layers are rearranged, respectively, followed by deleting the tool path with the step distance of $\mathrm{f}$. In a single-line tool path, the tool angle is changed and returned along the original tool path post-cutting. The sequence of the parity layer passes is highlighted in Fig. 7b, c, respectively. Increased cutting efficiency. First set a step size of $0.5 \mathrm{f}$. Not all tool paths get translated after cutting and editing the tool path. Improving the processing efficiency is still based on the precise tool path formed by the original surface.

The technical route of the CL sequence of the initial code is adapted as described in Fig. 8.

(1) Read the code line by line - The general idea of adapting the code is to use the " $f_{\text {open }}$ " function to open the initial code document and use the " $f$ getl" function to read the code line by line and form a string group. This is

Fig. 4 Processing parameters

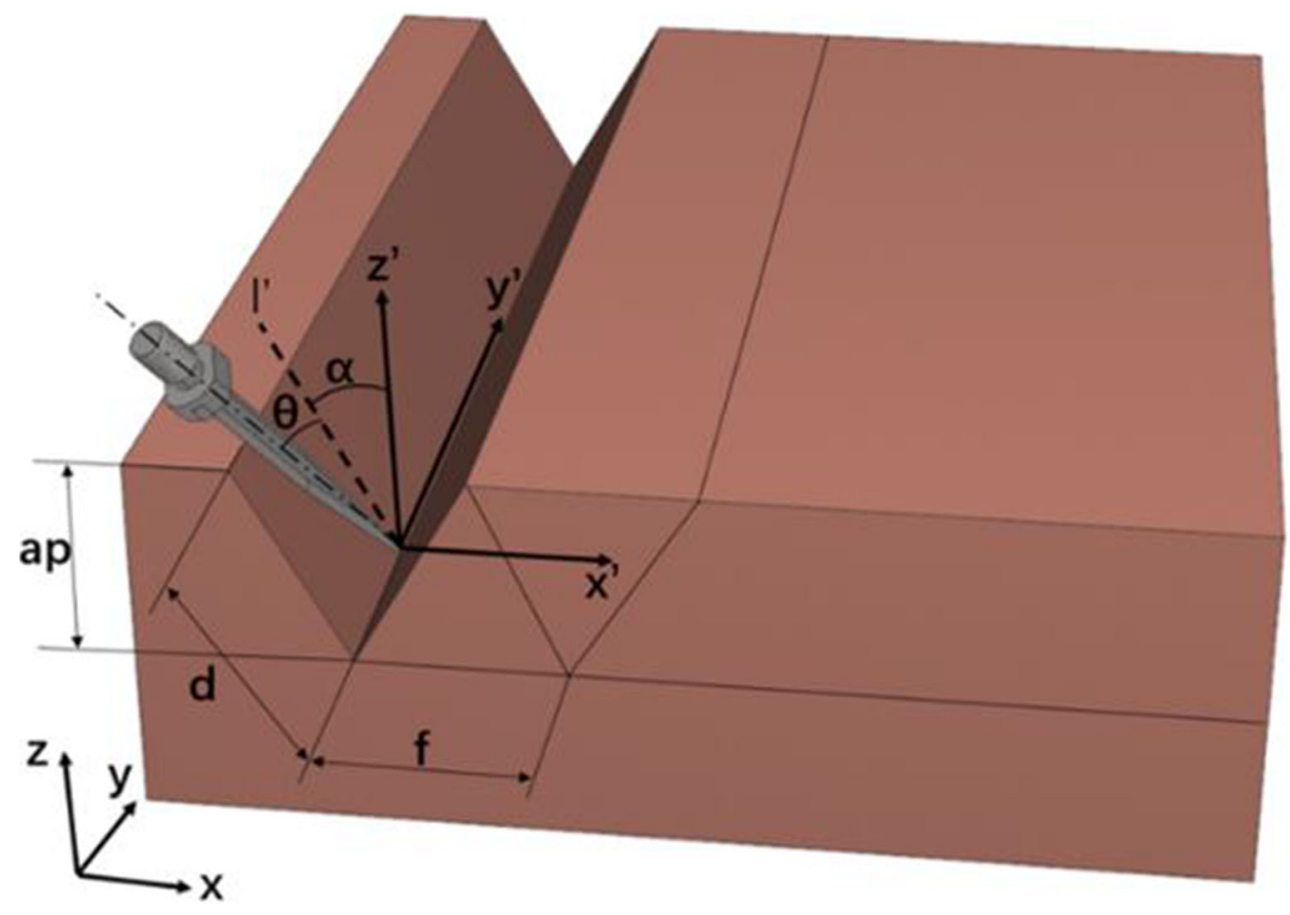




\section{equivalent cutter}

\section{disc cutter}

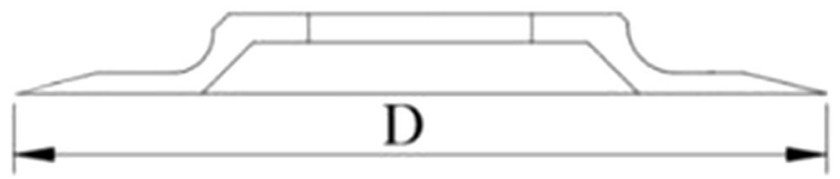

Fig. 5 Equivalent replace ultrasonic disc cutter

followed by identification, judgment, and adaptation of the content of each line of the string.

(2) Distinguish between odd and even layers-To distinguish the odd and even layers as an important preparatory link, the order of the CL of the odd and even layers must be adapted separately. The infeed is indicated by "EngageMove." The Z-coordinate of the infeed first appears in the next line of this mark. This coordinate represents the height of the current cutting layer in the Zdirection. With the change of this $Z$-coordinate value, it is feasible to distinguish the parity layer.

(3) Record the cutter counting location (CCL) - As described in Fig. 9, considering that the $X$-axis direction is the cutting direction, the presence of only the $X$-coordinate in the current $\mathrm{CL}$ indicates a new cutting tool path in the cutting order. This CL is recorded as CCL. Owing to the complex surface problem, not every tool path is a straight line along the $X$-axis. When the tool path is close to the curved surface, there is more than one CL with only $X$-coordinates in the same tool path, such as $x_{6}$.
During this time, the previous CCL in this tool path should be discarded and updated to the current CCL. As the turning mark of the tool path, CCL is primarily used to delete and adjust the order of CL.

(4) Delete and adjust the order of CL-The odd-numbered tool paths in the odd-numbered layers are reserved, and the odd-numbered tool paths in the even-numbered layers are deleted with the same deletion method. In the odd-numbered layers, odd-numbered tool paths need to be retained. After the end of the tool path, return along the original path and enter the next tool path. This process is started when the number of CCL is odd. At this point, record the point behind the CLs and write in reverse order when the CCL is even.

(5) Delete the duplicate CL-After adjusting the CL sequence, there will be a situation where the information of the current CL and the previous CL are overlapped. In this case, the current CL should be identified and deleted.

\subsection{Research on information planning of lifting and lowering the cutter}

The code generated by the preliminary planning based on the cavity milling process using UG contains only three-axis $\mathrm{CNC}$ information. Hence, no change in the angle of the rotation axis is required when the tool changes the tool path inside the part. This avoids the lifting of the tool path turning point within the lifting CL. Therefore, it is mandatory to add information on lifting and lowering the cutter, along with the places where the cutter needs to be lifted. The main situations that need to lift the cutter are mentioned in Fig. 10.

(1) The situation of continuous cutter lift-A schematic diagram of the tool lifting situation is represented in Fig. 11. The process from CL $P_{1}$ to CL $P_{2}$ is a non-cutting process that the tool needs to lift. Usually, such a situation arises when the tool path turns to the edge of the convexly curved surface area. Point $P_{1}$ contains only $X$ coordinate information, and its absolute value is less than the $X_{\max }$. The moving distance in the $Y$-direction from the points $P_{1}$ to $P_{2}$ is greater than the moving distance in

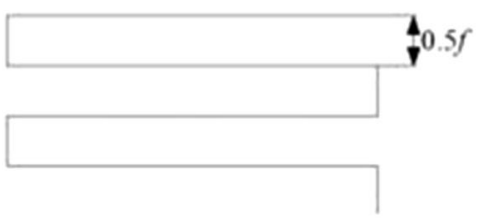

a

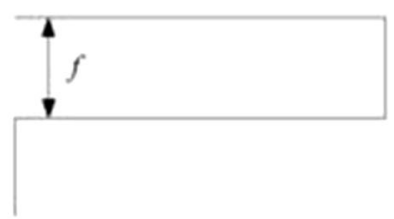

b

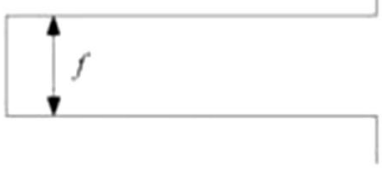

$\mathrm{C}$

Fig. 6 Sketch of tool path deletion 1 a. Before deletion. b. Odd-level tool path after deletion. $\mathbf{c}$ Even-level tool path after deletion 


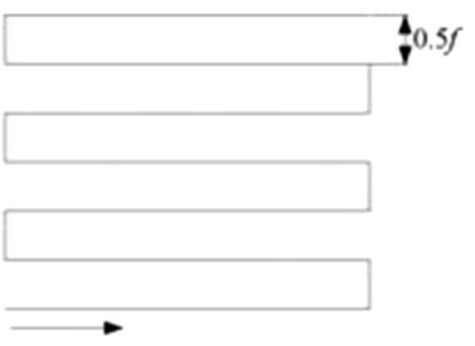

a

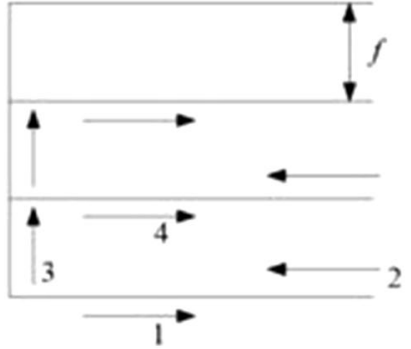

$\mathrm{b}$

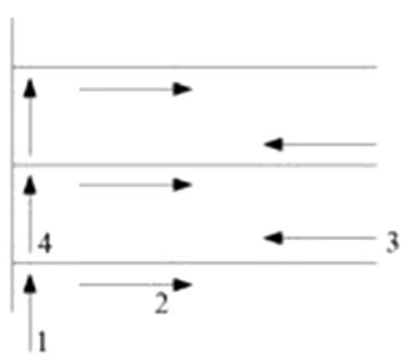

C

Fig. 7 Sketch of tool path deletion 2 a. Before deletion $\mathbf{b}$. Odd-level toolpath after deletion. c. Even-level toolpath after deletion

the $X$-direction. This indicates that the angle $\varphi$ between the stepping direction and the $X$-axis is greater than the limit value of the rotation angle of the straight blade cutter on the workpiece surface in the workpiece $\varphi_{\max }$. In this case, it is recognized that the cutter needs to be lifted at point $P_{1}$. The CL lift should be added after $P_{1}$, and the position of the lower cutter should be added after $P_{2}$. The next CL outside the blank should be added with the next cutter information. Since the process from the
CL $P_{1}$ to $P_{2}$ is a non-cutting process, the rotation angle information of the rotary axis before the point $P_{2}$ should be deleted while adding the tool lifting information. This boosts the work efficiency.

(2) The situation of area jump type cutter lifting. The CL P3 to $\mathrm{P} 4$ is the process from the normal complete cutting area to the incomplete cutting area with a convex curved surface. This process is shown in Fig. 11. This process does not require cutting, and the tool needs to be lifted
Fig. 8 The route of re-organizing the CL sequence

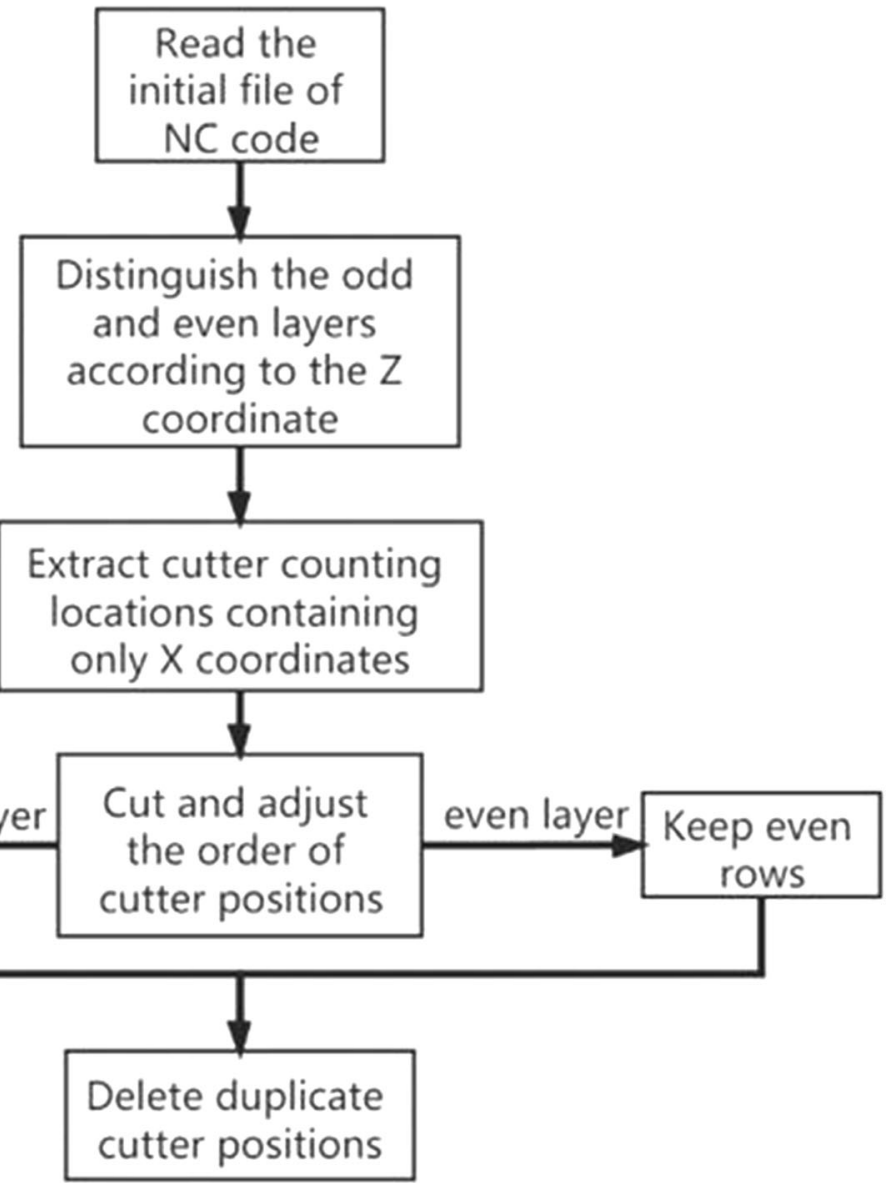


Fig. 9 The selection rule of CCL

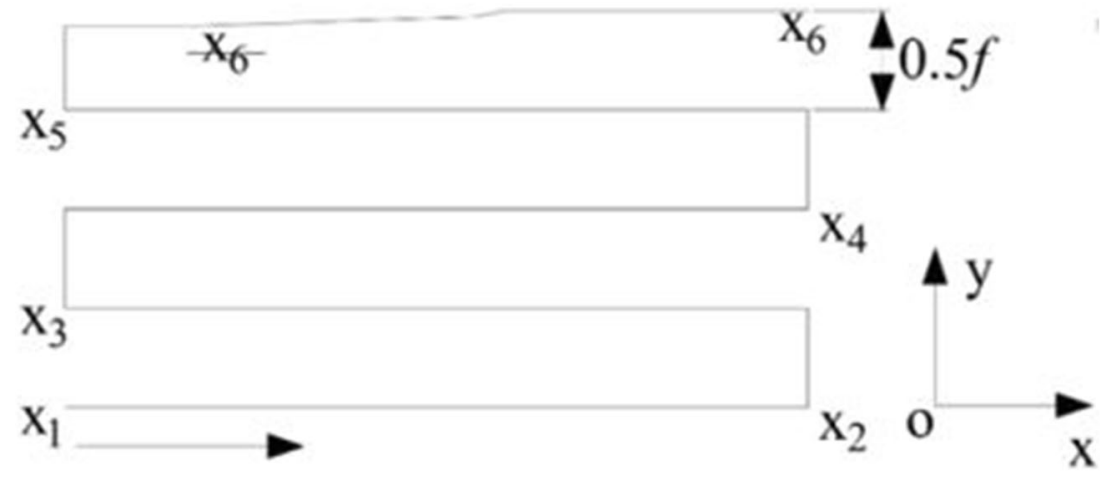

after point P3 to move towards point P4. The judgment method, in this case, is as follows: The point $\mathrm{P} 4$ contains both X-coordinate and Y-coordinate information, the absolute value of the $\mathrm{X}$-coordinate is less than $\mathrm{Xmax}$, and the movement in the $Y$-direction is greater than $0.5 \mathrm{f}$. This situation is different from the situation (1). Here, the need to lift the cutter at point $P_{4}$ is recognized. The cutter lifting position should be added before $P_{4}$, and the lower cutter position is added to $P_{4}$. When adding the tool lifting information, the rotation angle information is deleted before $P_{4}$ to improve the work efficiency.

(3) The tool path changes too much. It can be observed in the CLs $P_{5}, P_{6}$, and $P_{7}$ of Fig. 11 that when a convex curved surface affects the tool path planning of the current layer but does not block the entire tool path, a single tool path will detour. As shown in the tool path of the points $P_{5}$ to $P_{6}$, there will be a situation where the angle between the small tool path and the $X$-axis $\varphi$ changes too much. In this case, the absolute value of the $X$-coordinate of the point $P_{5}$ is less than $X_{\max }$, and the angle between $P_{5} P_{6}$ and the upper tool path exceeds $\varphi_{\max }$. The method of lifting the cutter, in this case, is the same as that in case (1). The amount of overcut generated by deleting the curved section in this tool path is very small (about 0.5 $\mathrm{mm}$ ) because the surface of aerospace parts is very smooth, and the rough machining process has a large margin. Nonetheless, this will not affect the finishing process and the final part's surface integrity.
Fig. 10 The route of lifting and lowering

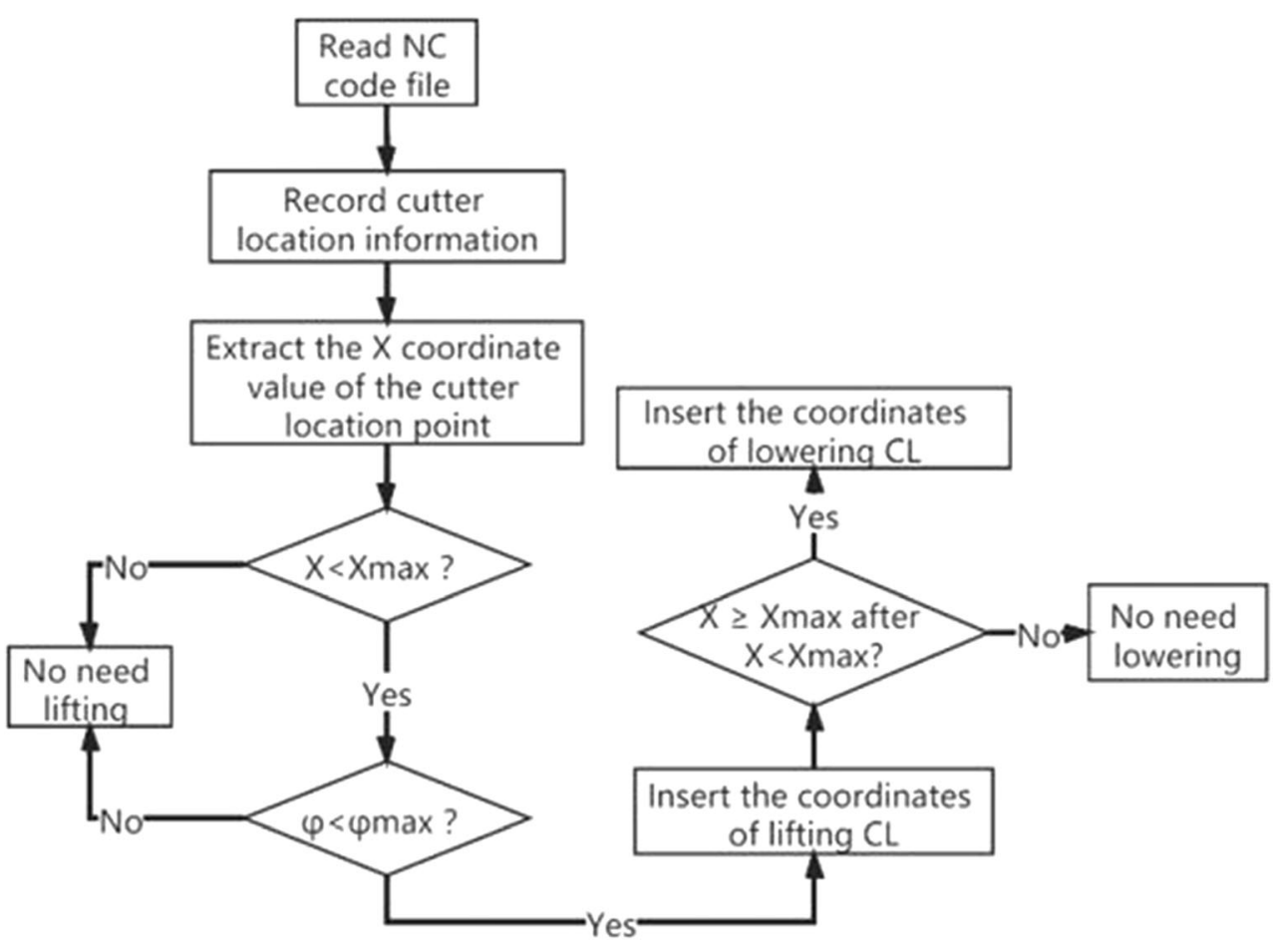


Fig. 11 "V" shape machining tool lifting

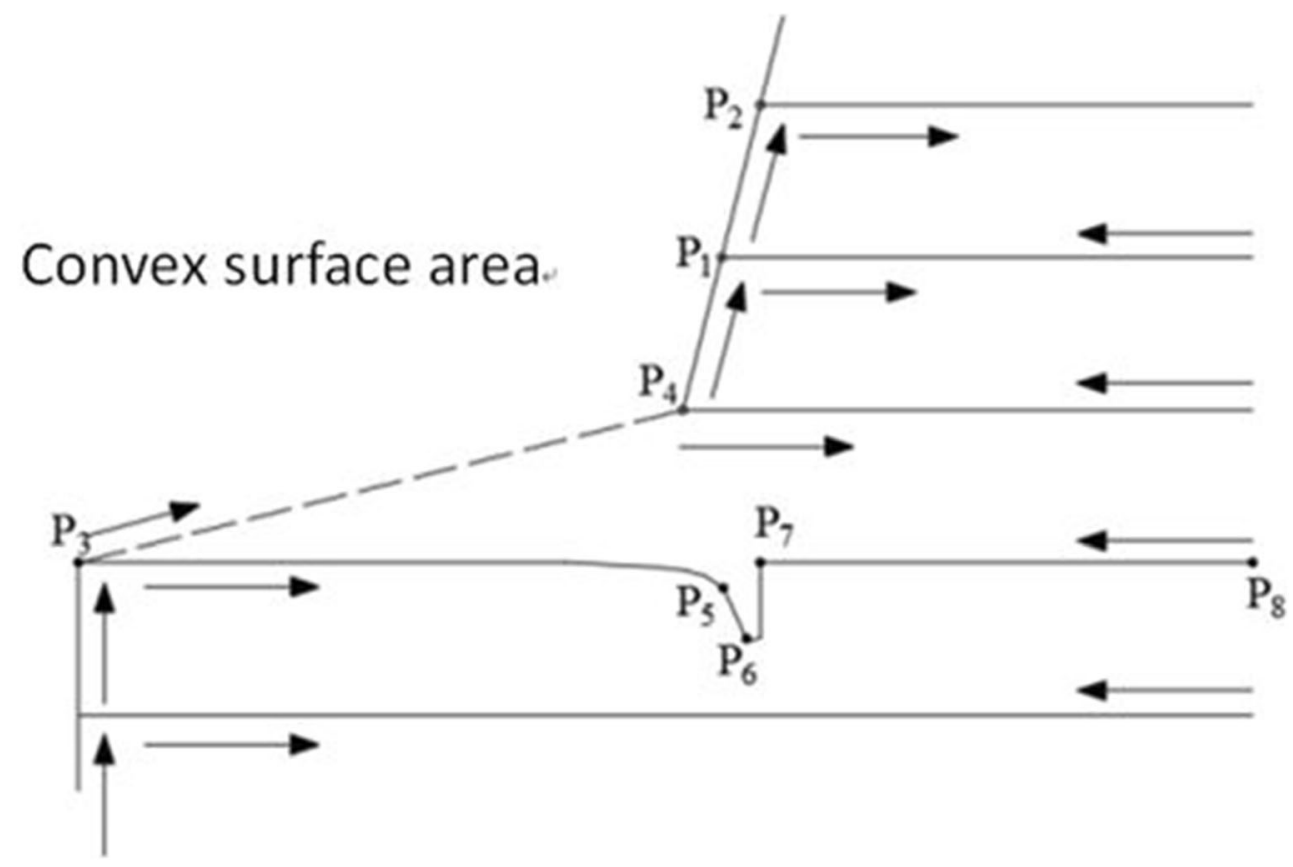

Among them, $X_{\max }$ indicates half of the maximum blank size in the $X$-direction of the blank set in $\mathrm{UG}$, and $\varphi_{\max }$ represents the limit value of the rotation angle of the straight blade cutter face in the workpiece.

\subsection{Research on the planning of chip breaking toolpath}

During the cutting process of "V" shape by using ultrasonic straight blade cutter as the processing tool, when the entire tool path does not encounter the inclined surface, the cutting needs to be done twice by the straight blade cutter to complete the chip removal. It can be inferred from Fig. 12 that for the inclined planes, the chips cannot fall off after the straight blade cutter has been cut back and forth along this path. This phenomenon is identified in this section, and a chip-breaking tool path has been added. The key steps are as follows:

(1) Record the cutter location of chip breaker (CLCB)-The position of the tool path that needs to be added with chip
Fig. 12 "V" machining process chip breaking

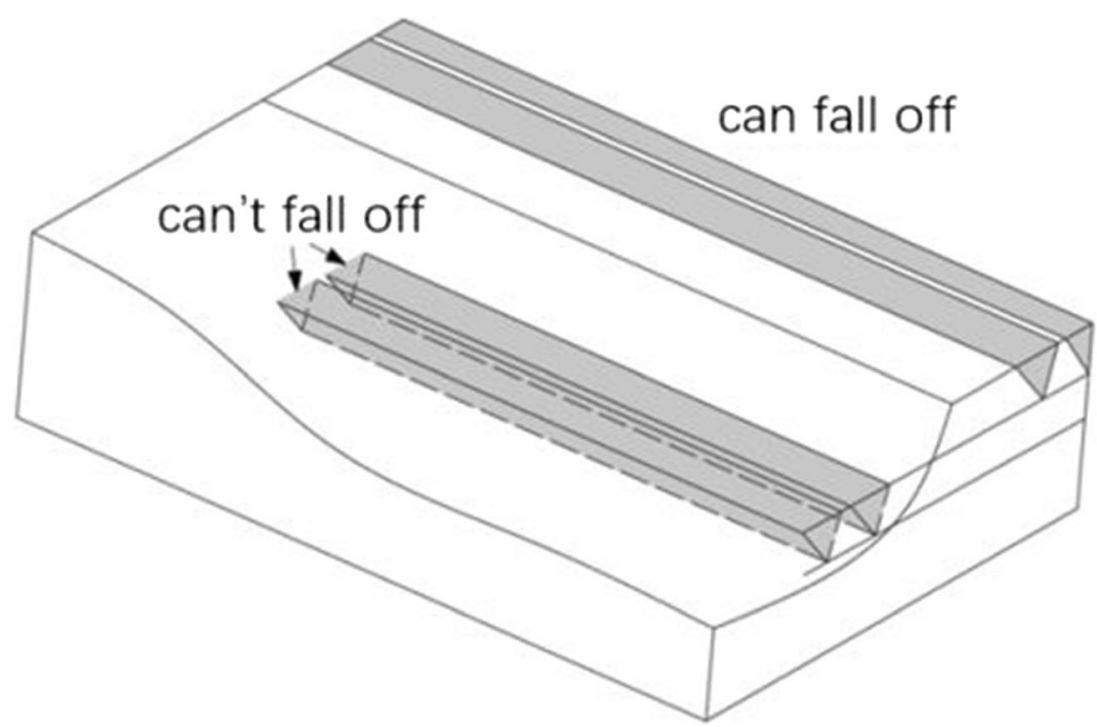


breaking is located at the tool lifting and lower CL studied in Section 3.2. Therefore, the CL with $Z$-coordinate information can be recognized by identifying the CL coordinate information or by determining the $X$ and $Y$ coordinate information of the current CL. If $x<x_{\max }$ is met and the difference of $Y$-coordinate with the previous CLCB is less than $0.5 \mathrm{f}$, the current $X$-coordinate and $Y$ coordinate information are recorded as the CLCB. The CLCB is used for subsequent connection to become a chip breaker tool path.

(2) Supplement the command angle for the CLCB-The blade posture of the straight blade cutter for cutting chips is not similar to that of the "V"-shaped machining process. The straight blade cutter only needs to include the forward inclination angle while cutting.

(3) Insert the CLC - By adding the information of each CL to the code in sequence, the chip-breaking tool path is formed. The specific method is as follows: If the CLCB appears in the current layer, the CLCB will be added after the "DepartureMove" sign appears at the end of the layer. A chip-breaking tool path is formed based on the CLCB.

(4) Raise the tool path to avoid interference of the tooltipBy raising the overall tool path, tooltip interference can be avoided.

\section{Cutting simulation experiment based on VERICUT}

The conventional code needs to be pre-coded, and the six-axis CNC machining code such as ultrasonic-assisted cutting must be simulated by the machine tool for performing the subsequent processes. For the aerospace manufacturing field, VERICUT is selected as the most optimum numerical control simulation processing software.

\subsection{Establishment of a motion simulation cutting model for a six-axis CNC machine tool}

\section{(1) Establish a six-axis gantry machine model}

Based on the processing module in the UG software, the initial model of the five-axis machine tool can be exported. Before importing into VERICUT, each axis of the model must be adapted. The ultrasonic tool holder (sixth axis) needs to be imported after the manual modeling.

\section{(2) VERICUT sets key points}

(a) Machine tool control system selection - Consistent with the post-processor in UG, 840D controller is also selected in the VERICUT. The controller supports a six-axis CNC machining process.

(b) The affiliation of each entity module is determined. In the case of a six-axis machine tool with an ultrasonic tool holder, the ultrasonic tool holder is subordinate to the $A$ axis as the sixth axis (third rotation axis) of the machine tool. The angle of the ultrasonic tool holder is controllable, and the tool is included in the ultrasonic tool holder. The affiliation of the remaining physical modules is such that the $A$-axis belongs to the $C$-axis, the $C$-axis belongs to the $Z$-axis, the $Z$-axis belongs to the $X$-axis, the $X$-axis belongs to the $Y$-axis, and the $Y$-axis belongs to the nonmovable machine tool body.

(c) Equivalent setting of the ultrasonic cutter-The formation process of the cutting effect in VERICUT is as follows: The part of the rotary tool passing through the workpiece disappears, and the straight blade cutter turns into a flat dagger cutter. During the simulation process, the use of an equivalent cylindrical milling cutter leads to the "V" machining process simulation process. Diamond chips cannot be displayed as chip loss. Therefore, the addition of a simulated chip removal tool path after the end of each cutting layer is mandatory. The tool is set to a tapered milling cutter, and the cone angle is the swing angle in the "V"-shaped machining process.

(d) Angle range setting - The control mode of the rotary axis must be set to the shortest distance. This avoids the small rotation of the tool during the cutting process (such as -1 to $0^{\circ}$ to $1^{\circ}$ ) into a large rotation (such as -1 to $180^{\circ}$ to $1^{\circ}$ ).

(e) Set comparison parts-VERICUT can export a complete and detailed process report and has an automatic comparison function. Hence, the addition of comparative parts will effectively assist in the study of the feasibility of the adapted code.

\subsection{Machine tool simulation and inspection of case parts}

For machine tool motion simulation, the simulation code modified post-processing is imported into VERICUT. During the process planning, the maximum cutting depth is set to $7.66 \mathrm{~mm}$, and the step distance is $12.3 \mathrm{~mm}$. No interference is observed between the tool holder and the workpiece during the motion simulation process. Fig. 13 highlights the rough machining simulation results. No over-cutting is noticed in the tip of the "V"-shaped tool path. A small amount of over-cutting is done to cut off the tool path to ensure smooth chip removal during cutting. The residual height is not correctly measured by VERICUT due to the huge gap between the residual entity and the traditional milling residual entity after the end of the "V" machining process. The residual height is sampled and measured in the software, and the 
Fig. 13 Rough machining residual solid model

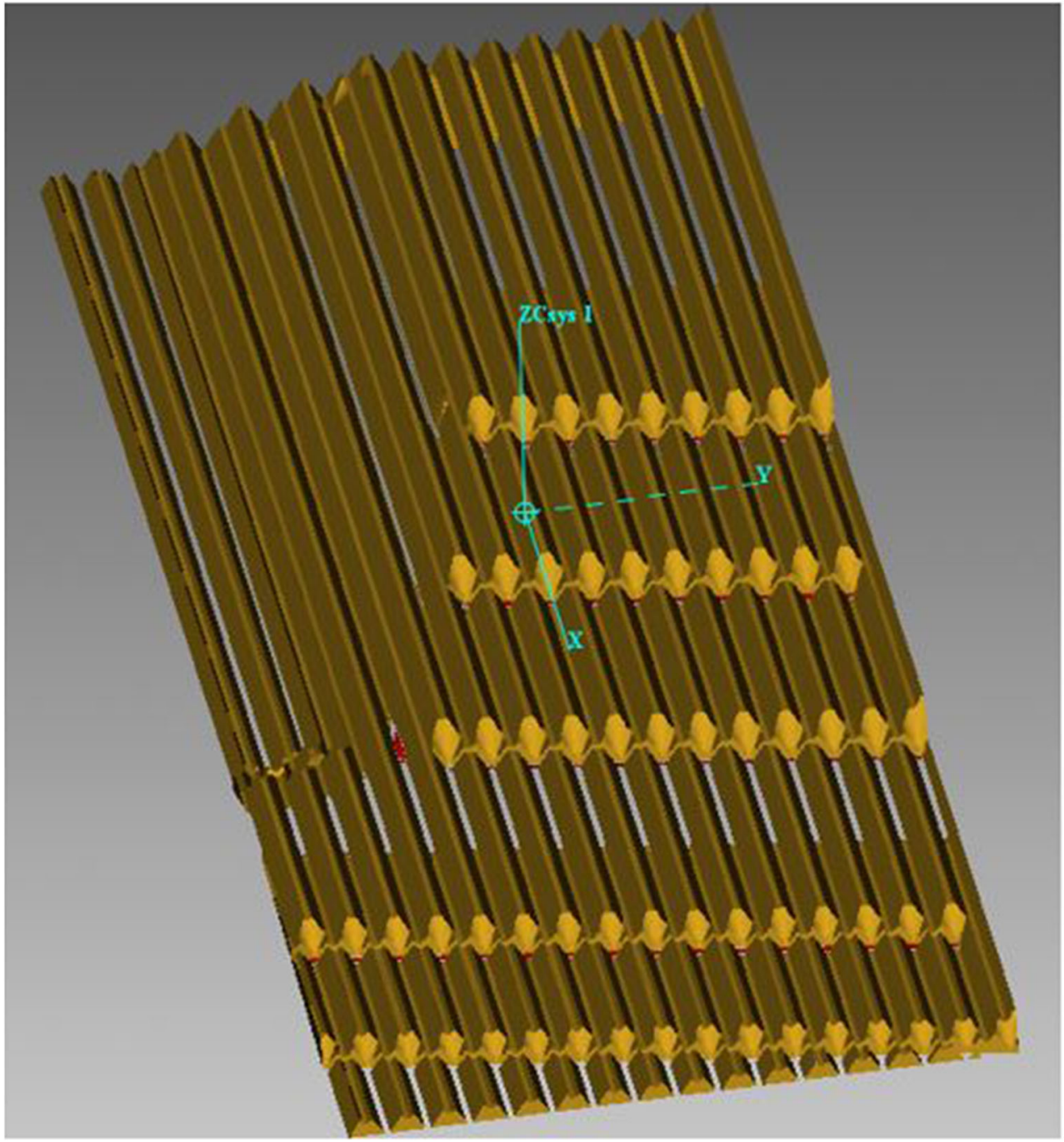

maximum recorded residual height is less than $10 \mathrm{~mm}$. The distance from the top of most diamond chips to the curved surface is less than $8.5 \mathrm{~mm}$. This meets the requirements of finishing.

The disc cutter is equivalently replaced with the cylinder milling tool of similar diameter, and the finishing code generated is imported into VERICUT. During the process planning, the maximum residual height is set to $0.2 \mathrm{~mm}$. The spindle and the workpiece do not interfere during the motion simulation process. The simulation results of the finishing process are displayed in Fig. 14. These results are consistent with the cutting results of the pre-code simulation performed in the UG software.

The motion simulation process and results of the " $\mathrm{V}$ " machining technology machine tool show that the post-processed rough machining code meets the ultrasonic cutter processing standards and can be used in the six-axis CNC gantry machining center. It can be inferred from the finishing results that the process planning method based on ultrasonic disc cutter for complex areas divided into regions is feasible, and the processing results meet the expected standard.

\section{Conclusions}

For ultrasonic-assisted processing of honeycomb cores, this research proposes a post-processing method of CNC machining. The "V" machining process is first selected, and then a preliminary process planning of the " $\mathrm{V}$ " machining process is made using UG. The $840 \mathrm{D}$ post-processor is used to generate a preliminary three-axis code, and the finishing process is directly replaced by the tool to design a machinable code. Secondly, the code is post-processed based on the technological characteristics of the ultrasonic straight blade cutter in the "V" machining process. This generates a six-axis code containing motion information of the ultrasonic tool holder. Three 
Fig. 14 Comparison model of finishing residual entities and case parts

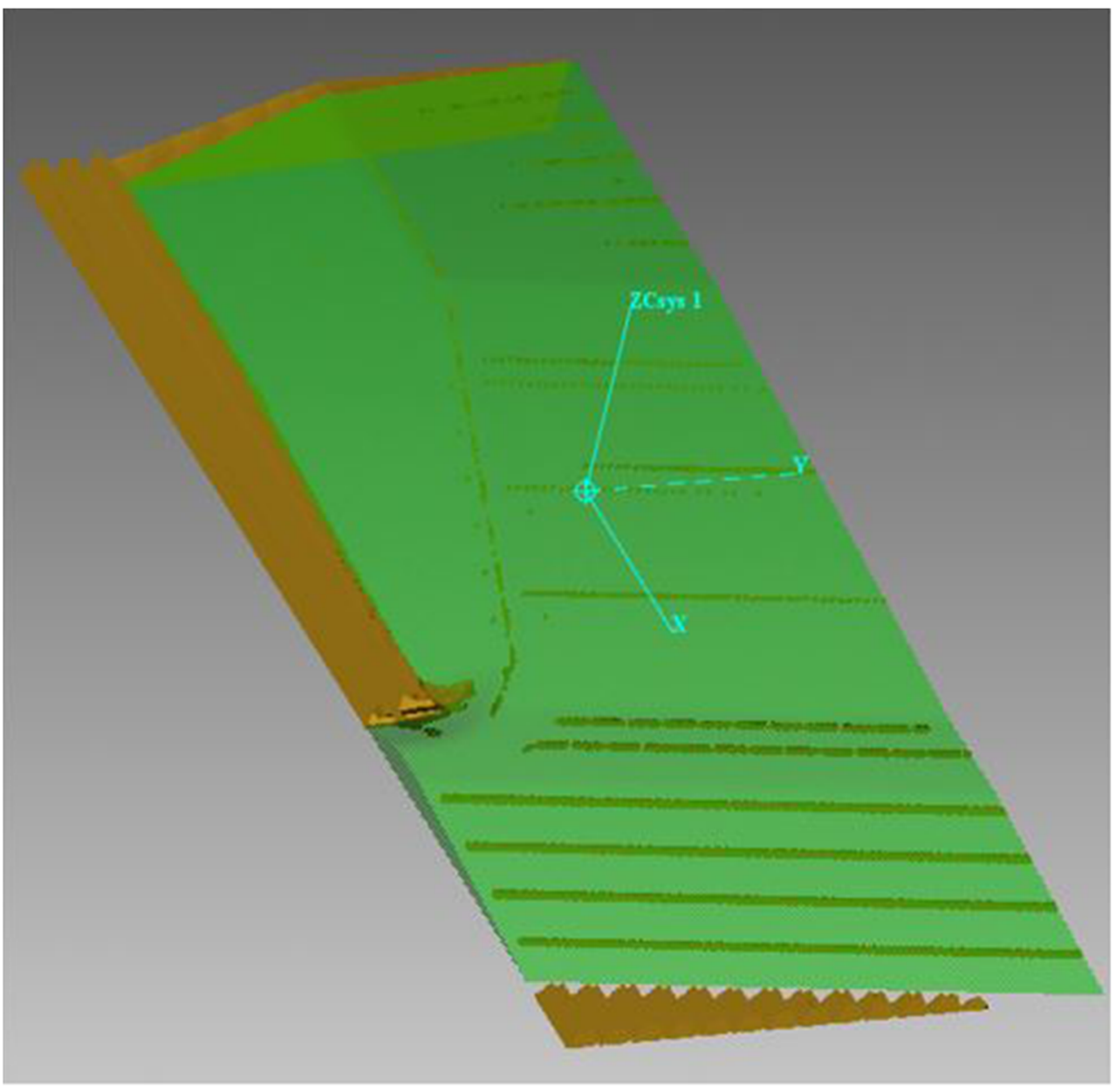

key technologies used are the second arrangement of CLs, research on information planning of lifting and lowering cutter, and research on planning of chip breaking toolpath. Eventually, the feasibility of the code processed by the postprocessing module is confirmed through the VERICUT sixaxis $\mathrm{CNC}$ machine tool motion simulation.

Author contribution Xiaoping Hu and Baohua Yu planned the research. Haofeng $\mathrm{Yu}$ and Dongfang $\mathrm{Mu}$ did experiments and analyzed the data. Dongfang $\mathrm{Mu}$ wrote the paper.

Funding This study was funded by the National Natural Science Foundation of China (No.51975173).

Data availability This article contains all the data gathered or analyzed during this study.

\section{Declarations}

Ethics approval and consent to participate None of the studies mentioned in this article contain any human participation. Also, no animals were harmed during these experiments. The authors consent to participate.
Consent for publication The authors provide their consent to publish this article.

Competing interests The authors declare no competing interests.

Open Access This article is licensed under a Creative Commons Attribution 4.0 International License, which permits use, sharing, adaptation, distribution and reproduction in any medium or format, as long as you give appropriate credit to the original author(s) and the source, provide a link to the Creative Commons licence, and indicate if changes were made. The images or other third party material in this article are included in the article's Creative Commons licence, unless indicated otherwise in a credit line to the material. If material is not included in the article's Creative Commons licence and your intended use is not permitted by statutory regulation or exceeds the permitted use, you will need to obtain permission directly from the copyright holder. To view a copy of this licence, visit http://creativecommons.org/licenses/by/4.0/.

\section{References}

1. Toozandehjani M, Kamarudin N, Dashtizadeh Z, Lim EY, Gomes A, Gomes C (2018) Conventional and advanced com-posites in aerospace industry: Technologies Revisited. Am J Aerosp Eng 5(1):9 
2. Karakoi A, Freund J (2012) Experimental studies on mechanical properties of cellular structures using Nomex ${ }^{\circledR}$ honeycomb cores. Compos Struct 94(6):2017-2024

3. Kang D, Zou P, Wu H, Duan J, Wang W (2019) Study on ultrasonic vibration-assisted cutting of Nomex honeycomb cores. Int J Adv Manuf Technol 104(1-4):979-992

4. Jaafar M, Atlati S, Makich H, Nouari M, Moufki A, Julliere B (2017) A 3d fe modeling of machining process of Nomex honeycomb core: influence of the cell structure behaviour and specific tool geometry. Procedia Cirp 58:505-510

5. Pan XL (2019) Four-axis complete machining process planning and tool path post-processing of complex curved impeller. Master's degree thesis of Xi' an Shiyou University

6. Li J, Zhu XX (1996) General post-processing system introduction. Digital Manufacturing Industry (6):19-21 (in Chinese)

7. Li LJ, Sun W (2014) Research of postprocessor for Heidenhain dual-table five-axis machine based on UGNX6.0. Machine Tool \& Hydraulics 42(10):122-125 (in Chinese)

8. Li TG (2014) Research on post processing for five-axis machining tool based on Edgecam. Machine Tool \& Hydraulics 42(20):4-6 (in Chinese)

9. Hao QM, Jiang J (2015) The customization and simulation about the double pendulum station of five-axis post-processing based on
MasterCAM. Manufacturing Technology \& Machine Tool (4): 176-79 (in Chinese)

10. Yu DY, Ding Z (2019) Post-processing algorithm of a five-axis machine tool with dual rotary tables based on the TCS method. Int J Adv Manuf Technol 102:3937-3944

11. Wang Y, Kang R, Dong Z, Wang X, Zhang X (2021) A novel method of blade-inclined ultrasonic cutting Nomex honeycomb core with straight blade. J Manuf Sci Eng 143(4):1-34

12. Cui R, Zhang J, Feng P, Yu D, Wu Z (2020) A path planning method for $\mathrm{v}$-shaped robotic cutting of Nomex honeycomb by straight blade tool. IEEE Access PP 99:1-1

13. Huang XX, Hu XP, Yu BH, Wu SY (2015) Ultrasonic cutting mechanism of Nomex honeycomb composites based on fracture mechanics. J Mech Eng 51(23):205-212

14. Yu HF, Hu XP, Kong LY, Yu BH (2019) Process path planning based on efficiency model for ultrasonic cutting curved surface of honeycomb composite parts. Adv Mech Eng 11(10):1-11

Publisher's note Springer Nature remains neutral with regard to jurisdictional claims in published maps and institutional affiliations. 